\title{
Fractionation of Poly(vinyl chloride)s by Preparative Scale Gel-Permeation Chromatography
}

\author{
Shigeru Hattori, Hideyo ENDoH, Hisae NAKahara, \\ Toshio Kamata, and Motome Hamashima* \\ National Chemical Laboratory for Industry, 1-5, Honmachi 1-chome, \\ Shibuya-ku, Tokyo 151, Japan.
}

(Received July 5, 1977)

\begin{abstract}
Three poly(vinyl chloride) (PVC) samples were fractionated by a preparative-scale GPC (gel-permeation chromatography) with Styragel. The carrier solvent used was tetrahydrofran. The fractionation of one sample whose average molecular weight was the lowest among the three samples was not affected much by the injected sample concentration and all the fractions obtained gave fairly narrow distributions of molecular weight. The fractionation of the remaining two samples having higher average molecular weights showed an overload effect and the polydispersity of each fraction of these samples was greater than that of the former sample. The relationship between the intrinsic viscosity and weightaverage molecular weight for PVC fractions revealed that the fractions of the latter two samples contained long chain branches. The GPC calibration curve for the hydrodynamic volume of PVC agreed with that of the polystyrene standards in the low molecular-weight range, but not in the high molecular-weight range where the effect of chain branching was dominant.

KEY WORD Preparative Scale GPC/Poly(vinyl chloride)/ Tetrahydrofuran / Overload Effect / Polydispersity / Branching / Universal Calibration /
\end{abstract}

Narrow molecular weight distribution polymers are frequently required as standard samples for measuring various physical properties. Narrowmolecular-weight-distribution polystyrenes can be prepared by living polymerization, and such polystyrenes have already been placed on the market and used widely. Today poly(vinyl chloride) (PVC) is commercially produced in the largest quantity among a variety of polymers, and so PVC standard samples are also needed. However, at the present time fractionation is the only method available for the preparation of narrow-molecular-weight-distribution PVC, since the living polymerization method is not applicable to PVC.

The fractionation of polymers by the preparative-scale GPC (gel-permeation chromatography) has been attempted with several polymers, ${ }^{1-11}$

* Present address: Department of Environmental Chemistry, Faculty of Engineering, Utsunomiya University, 2753, Ishii-cho, Utsunomiya 321-31, Japan. but a report by Peyrouset, et al.,$^{6}$ is the only example reported in regard to PVC. They fractionated a PVC sample with a weight-average molecular weight $\left(M_{v}\right)$ of $8.97 \times 10^{4}$, using silica beads (Spherosil) and obtained fractions with a polydispersity (the ratio of $M_{w}$ to number-average molecular weight $M_{n}$ ) of $1.1-1.2$. The National Physical Laboratory of England has recently placed on the market a series of PVC fractions obtained by the preparative-scale GPC as PVC standard samples. However, the polydispersity of these samples ranges from 1.4 to $1.6^{12}$ but is not as small as is desirable.

The authors previously undertook the fractionation of polystyrene by means of preparative scale $\mathrm{GPC}^{3}$ to investigate experimental conditions for obtaining narrow-molecular-weight-distribution fractions. Likewise, poly(methyl methacrylate) ${ }^{11}$ was fractionated and fairly narrow-molecularweight-distribution samples were obtained. In this work, the authors attempted to fractionate PVC by the preparative scale GPC into com- 


\section{S. Hattori, H. Endoh, H. Nakahara, T. Kamata, and M. Hamashima}

ponents having a sufficiently narrow molecularweight distribution that is usable as standard samples and have determined what conditions would be necessary for such fractionation.

\section{EXPERIMENTAL}

\section{Materials}

Three kinds of PVC samples were used: sample A which has the highest average molecular weight among the three samples was prepared on a laboratory scale; sample $\mathrm{B}$, a commercially available material, and while sample $C$ which is a lowmolecular-weight fraction obtained from another commercial material by precipitation fractionation.

Commercial tetrahydrofuran (THF) of extra pure grade (stabilized with $c a .0 .025 \%$ of butylated cresol) was used without further purification for both the preparative scale and the analytical GPC. THF used for light scattering and viscosity measurements was a guaranteed grade reagent and distilled immediately before use. Cyclohexanone of a guaranteed grade was used without further purification for osmometry.

\section{The Preparative Scale and the Analytical GPC}

An apparatus manufactered by Waters Associates, Inc., ANA-PREP GPC, was used for the preparative scale GPC. Three preparative columns, 2 inches in diameter, 4 feet in length and packed with Styragel (indicated pore size, $3 \times 10^{6}$, $10^{5}$, and $10^{4} \AA$ ), were used for fractionation. THF was used as the carrier solvent and the experimental conditions in all cases were: temperature, $35^{\circ} \mathrm{C}$; the volume of injected solution in each run, $150 \mathrm{ml}$; flow rate, $30 \mathrm{ml} / \mathrm{min}$. The other conditions are shown in Table I. Each fraction in THF solution was concentrated by a

Table I. Fractionation of PVC

\begin{tabular}{ccccc}
\hline $\begin{array}{c}\text { Experi- } \\
\text { mental } \\
\text { Code }\end{array}$ & Sample & $\begin{array}{c}\text { Concen- } \\
\text { tration of } \\
\text { injected } \\
\text { solution, } \\
\mathrm{g} / \mathrm{d} l\end{array}$ & $\begin{array}{c}\text { Number } \\
\text { of } \\
\text { injection }\end{array}$ & $\begin{array}{c}\text { Volume of } \\
\text { collected } \\
\text { solution of } \\
\text { one fraction, } \\
\mathrm{m} l\end{array}$ \\
\hline A-1 & $\mathrm{A}$ & 0.5 & 8 & 300 \\
$\mathrm{~A}-2$ & $\mathrm{~A}$ & 0.2 & 1 & 300 \\
$\mathrm{~A}-3$ & $\mathrm{~A}$ & 0.2 & 1 & 150 \\
$\mathrm{~B}-1$ & $\mathrm{~B}$ & 0.5 & 8 & 300 \\
$\mathrm{~B}-2$ & $\mathrm{~B}$ & 0.2 & 1 & 300 \\
$\mathrm{C}-1$ & $\mathrm{C}$ & 0.5 & 8 & 300 \\
$\mathrm{C}-2$ & $\mathrm{C}$ & 0.2 & 1 & 300 \\
\hline
\end{tabular}

rotary evaporator and the PVC was precipitated by the addition of methanol. The PVC fraction thus precipitated was filtered and dried thoroughly in a vacuum drier.

A Toyo Soda HLC-801A liquid chromatograph was used with two TSK-GMH6 columns ( 2 feet $X$ 2) for analytical GPC measurements under the following conditions: solvent, THF; temperature, $40^{\circ} \mathrm{C}$; injected sample concentration, $0.1 \mathrm{~g} / \mathrm{d} l$; the volume of injected solution, $0.5 \mathrm{ml}$; flow rate, $1.2 \mathrm{ml} / \mathrm{min}$. The height of chromatogram was measured every at $1 / 4$ count (one count represents 100 drops or an elution volume of $c a .1 \mathrm{ml}$ ) in order to determine $M_{w} / M_{n}$.

\section{Characterization}

Light-scattering measurements were made on a Shimadzu PG-21 light-scattering photometer in THF at $20^{\circ} \mathrm{C}$ with unpolarized light of $435.8 \mathrm{~nm}$ in wavelength. The refractive index increment, $\mathrm{d} n / \mathrm{d} c$, was $0.115 \mathrm{ml} / \mathrm{g}$ (observed value). Optical clarification was carried out by centrifuging the sample solution at $60,000 \mathrm{G}$ for about $1 \mathrm{hr}$ on a Spinco L-4 preparative ultracentrifuge and then filtering the solution through a Corning ultrafine glass filter. The weight-average molecular weight of each of the three unfractionated samples was determined by the Zimm plot and those of the fourteen fractions were determined in accordance with the one concentration approximation method. ${ }^{13}$ In the latter case, corrections were made by taking the second virial coefficient as equal to $1.0 \times 10^{-3} \mathrm{~mol} \mathrm{~cm} / \mathrm{g}^{2}$.

Osmotic pressure was determined on a Mechrolab 502 high spped membrane osmometer in cyclohexanone at $37^{\circ} \mathrm{C}$. The membrane used was the Schneichel \& Schnell O-8.

Viscosity was measured on a FICA's automatic capillary viscometer, VISCOMATIC, in THF at $35^{\circ} \mathrm{C}$. Corrections for kinetic energy were made in determining the intrinsic viscosity $[\eta]$.

\section{RESULTS AND DISCUSSION}

Figure 1 presents the plot of the weight against the fraction number obtained in the experiments on fractionation. Each fraction number on the abscissa represents an elution volume of $300 \mathrm{ml}$. In Figure 1, the solid lines refer to the cases of $0.5 \mathrm{~g} / \mathrm{d} l$ in the injected sample concentration, i.e., experimental code $\mathrm{A}-1, \mathrm{~B}-1$, and $\mathrm{C}-1$ in Table $\mathrm{I}$ 


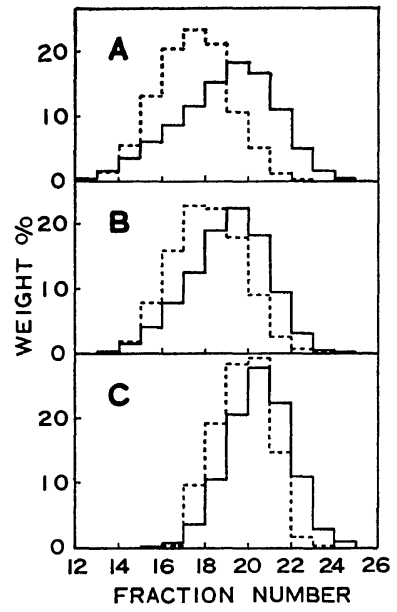

Figure 1. Results of the fractionations of PVC. Injected sample concentrations:,$- 0.5 \mathrm{~g} / \mathrm{d} l$; -----, $0.2 \mathrm{~g} / \mathrm{d} l$.

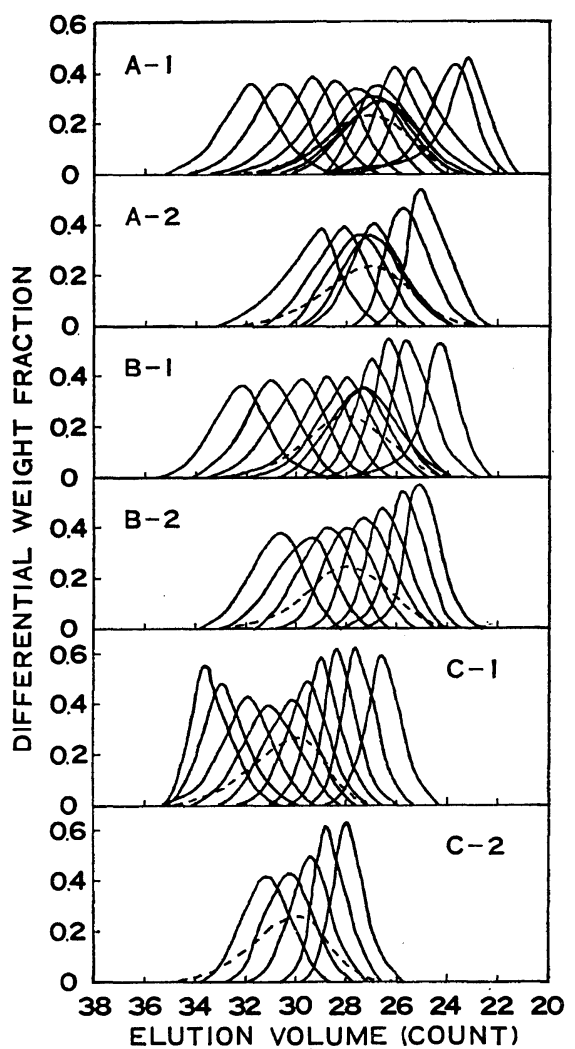

Figure 2. Analytical gel permeation chromatograms for PVC fractions (-) and unfractionated samples (----).

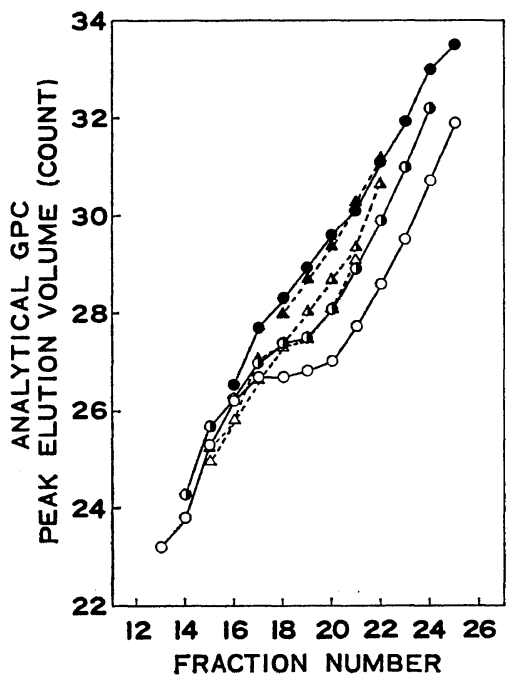

Figure 3. Relationship between analytical GPC peak elution volume and fraction number: $\mathrm{O}, \mathrm{A}-1 ; \mathbf{O}, \mathrm{B}-1$; $\bullet, \mathrm{C}-1 ; \triangle, \mathrm{A}-2 ; \mathbf{\Delta}, \mathrm{B}-2 ; \mathbf{\Delta}, \mathrm{C}-2$.

while the dotted lines refer to the cases of $0.2 \mathrm{~g} /$ $\mathrm{d} l$, i.e., the experimental code $\mathrm{A}-2, \mathrm{~B}-2$, and $\mathrm{C}-2$. As in Figure 1, the elution of the sample as a whole tends to occur faster as the injected sample concentration is lowered. In particular, this tendency becomes more pronounced as the average molecular weight of the sample is increased. This phenomenon is well known as the overload effect in analytical GPC.

The fractions thus obtained were then subjected to the anlytical GPC with the exception of such fractions too small in quantity to permit analyzing. The chromatograms are shown in Figure 2 where the dotted lines represent the chromatograms of the unfractionated samples. The relationship between the analytical GPC peak elution volume and the fraction number is presented for the fractions in Figure 3. In any one of the fractions up to No. 16 , the peak elution volume of a given fraction number does not differ very much from one fractionation experiment to another. Above No. 17, however, the relationship between the peak elution volume and the fraction number shows a considerably different tendency for each fractionation. In experiment $\mathrm{A}-1$, the peak elution volume undergoes virtually no change from fraction No. 17 to N0. 20 and the chromatograms of those fractions nearly overlap. This indicates that the high concentration portions are not separated 


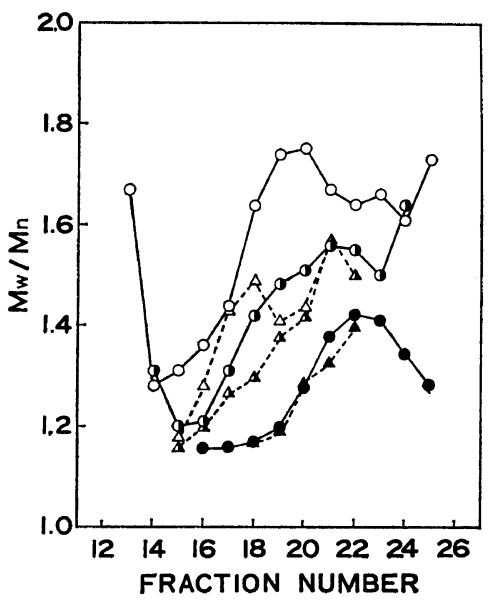

Figure 4. Relationship between $M_{w} / M_{n}$ calculated from analytical GPC and fraction number. Symbols are the same as shown in Figure 3.

sufficiently owing to the overload effect and the elution of such portions is delayed markedly. This delay in elution affects the elution of the lowmolecular-weight fractions. So the peak elution volumes of the lower-molecular-weight fractions in A-1 are considerably smaller than those of other experiments when compared at the same fraction number. Experiments A-2 and B-1 show roughly the same relationship. In these experiments, the delay in elution as a whole becomes fairly small, but some delay in elution is still noted near the fraction No. 18. This delay becomes virtually unrecognizable in experiment $\mathrm{B}-2$. On the other hand, sample $C$ shows essentially the same relationship between the peak elution volume and the fraction number even when the injected sample concentration is varied and it shows no delay in elution such as noted in either sample A or B.

The relationship between the ratio $M_{w} / M_{n}$ obtained from the analysis of the chromatograms and the fraction number is shown in Figure 4. In such an analysis, the relationship between the molecular weight and the elution volume (GPC calibration) for the standard polystyrene samples (prepared by Toyo Soda and Pressure Chemical) was used directly. As shown in Figure 4, the ratio $M_{w} / M_{n}$ is relatively small for those fractions eluted at an early stage, except for the first fraction in experiment A-1 and B-1, and increases as the fraction number goes up, to the point where the largest elution of the sample occurs (for example, fraction
No. 20 in experiment A-1); thereafter, it changes very little or tends to decrease slightly. Moreover, the comparison of $M_{w} / M_{n}$ at the same fraction number reveals the effects of the molecular weight of the injected sample and the injected sample concentration. That is, each fraction of A-1 shows the largest $M_{w} / M_{n}$ followed by the corresponding fractions of A-2, B-1, and B-2. Each fraction of the sample $\mathrm{C}$ shows the smallest $M_{w} / M_{n}$ and is practically free from any effect of the injected sample concentration. As for the overload effect in preparative scale GPC such as observed here, the similar results have been reported on polystyrene $^{3,7,8}$ and poly(methyl methacrylate). ${ }^{11}$

Next, in experiment A-3, the sample $A$ was fractionated under the same conditions as for A-2 except that the volume of collected solution of a fraction was made $150 \mathrm{ml}$; that is, one half of that in A-2. In this case, since the apparatus used in this work could not be used to collect automatically a fraction of less than $300 \mathrm{ml}$ in volume, the fractions were collected manually. The ratio $M_{w} / M_{n}$ of the fractions thus collected is plotted together with $M_{w} / M_{n}$ of the fractions in A-2 in Figure 5. It is seen that halving of the volume of collected solution somewhat diminishes the $M_{w} / M_{n}$ of a fraction in the early stage of elution, but affects very little the $M_{w} / M_{n}$ of the fractions above No. 19 . It seems likely that the reduction of the collected solution volume of a fraction is effective to some extent for reducing the $M_{w} / M_{n}$ of the high-molecular-weight fractions but not very effective for the low-molecular-weight fractions. This may be due to the disturbance of the

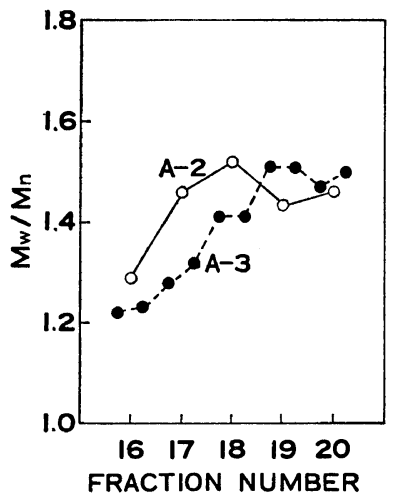

Figure 5. Effect of the collected solution volume of a fraction on $M_{w} / M_{n}$ of the fraction. 
Fractionation of PVC by Preparative Scale GPC

Table II. Molecular weights, polydispersities, and intrinsic viscosities of PVC

\begin{tabular}{lccccc}
\hline $\begin{array}{c}\text { Sample } \\
\text { code }\end{array}$ & $M_{w} \times 10^{-4}$ & $M_{n} \times 10^{-4}$ & $\left(M_{w} / M_{n}\right)_{\mathrm{LS}, \mathrm{OSM}}$ & $\left(M_{w} / M_{n}\right)_{\mathrm{GPC}}$ & $\begin{array}{c}{[\eta]_{T \mathrm{H}}^{35^{\circ}}} \\
\mathrm{d} l / \mathrm{g},\end{array}$ \\
\hline Unfractionated & & & & & \\
UF-A & 38.0 & 9.29 & 4.09 & 2.56 & 1.655 \\
UF-B & 17.4 & 5.40 & 3.22 & 2.43 & 1.211 \\
UF-C & 3.56 & 2.37 & 1.50 & 2.18 & 0.514 \\
Fraction & & & & & \\
A-1-15 & 105.2 & - & - & 1.31 & 2.899 \\
A-1-17 & 37.3 & 22.9 & 1.63 & 1.44 & 2.144 \\
A-1-22 & 7.64 & - & - & 1.64 & 0.923 \\
B-1-16 & 33.3 & - & - & 1.21 & 2.202 \\
B-1-17 & 32.3 & 20.5 & 1.58 & 1.31 & 1.830 \\
B-1-18 & 17.8 & 15.1 & 1.18 & 1.42 & 1.486 \\
B-1-20 & 10.9 & 8.14 & 1.34 & 1.51 & 1.043 \\
B-1-21 & 6.28 & 4.65 & 1.35 & 1.56 & 0.802 \\
B-1-22 & 4.56 & - & - & 1.55 & 0.586 \\
C-1-19 & 5.98 & 5.55 & 1.09 & 1.20 & 0.768 \\
C-1-20 & 4.25 & 3.79 & 1.12 & 1.28 & 0.594 \\
C-1-21 & 2.74 & 2.52 & 1.09 & 1.38 & 0.469 \\
C-1-22 & 1.80 & - & - & 1.42 & 0.337 \\
C-1-23 & 1.36 & - & - & 1.41 & 0.243 \\
\hline
\end{tabular}

delay in elution being dominant in the lowmolecular-weight range.

In Table II, $M_{w}$ determined by light scattering, $M_{n}$ determined by osmometry, the ratio $\left(M_{w}\right)$ $\left.M_{n}\right)_{\mathrm{LS}, \text { osm }}$ and $[\eta]$ measured in THF at $35^{\circ} \mathrm{C}$ are given for the three unfractionated samples and the fourteen fractions. The ratio $\left(M_{w} / M_{n}\right)_{\mathrm{GPC}}$ obtained from the analytical GPC (Figure 4) is also given in Table II. Comparison of $\left(M_{w} / M_{n}\right)_{\mathrm{LS}, \text { osM }}$ and $\left(M_{w} / M_{n}\right)_{\mathrm{GPC}}$ indicates that the former is larger than the latter in each of the four samples, UF-A, UF-B, A-1-17 and B-1-17 while this relation is reversed in the remaining seven samples. It is known that $\left(M_{w} / M_{n}\right)_{\mathrm{GPC}}$ is generally obtained as somewhat larger than the actual $M_{w} / M_{n}$ on account of zone broadening in GPC. However, the above-mentioned four samples show the opposite results. This may be due to these samples having long chain branches as will be described later.

Of the remaining seven samples, the $\left(M_{w} / M_{n}\right)_{\mathrm{GPC}}$ is larger than $\left(M_{w} / M_{n}\right)_{\mathrm{LS}, \text { osM }}$ by $10-20 \%$, except for UF-C and C-1-21. This is probably due to zone broadening in the analytical GPC. Now, $\left(M_{w} / M_{n}\right)_{\text {GPO }}$ is larger than $\left(M_{w} / M_{n}\right)_{\text {LS , OSM }}$ by $45 \%$ in UF-C and by $27 \%$ in $\mathrm{C}-1-21$. This may be accounted for as follows: the two samples are low in molecular weight and their low-molecularweight portions may permeate through the membrane so that measurements of the osmotic pressure give results of seemingly larger values of $M_{n}$.

The log-log plots of [ $\eta$ ] against $M_{w}$ are shown in Figure 6. The slope diminishes in the range of $M_{w}>10^{5}$, indicating that the fractions in question are branched polymers. The following relation was obtained from the plots in the range of $M_{w}<$ $10^{5}$ :

$$
[\eta]=1.8 \times 10^{-4} M_{w}^{0.76}
$$

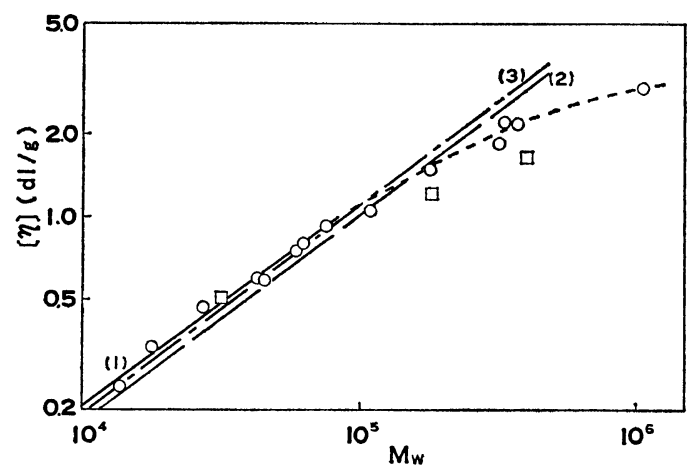

Figure 6. Log-log plot of $[\eta]$ vs. $M_{w}$ for PVC: $O$, fraction; $\square$, unfractionated sample; line (1) (-), this work $\left(M_{w}<10^{5}\right)$; line (2) $(---)$, Bohdanecký et al. ${ }^{15}$; line (3) (--), Freeman, et al. ${ }^{16}$ 
This corresponds to line (1) in Figure 6. Many studies are known concerning the relationship between [ $\eta$ ] and $M_{w}$ for PVC in THF. However, the results of these studies do not always show good agreement among themselves. Bohdanecký, et al., ${ }^{14}$ conducted a detailed study on this aspect and stated that it is most reasonable to assign a value of $0.75-0.80$ to the exponent of the $[\eta]$ $M_{w}$ equation for PVC in THF. For comparison, two equations for the relationship between [ $\eta]$ and $M_{w}$ in which the exponent is $0.75-0.80$ are shown in Figure 6. One such equation was derived by Bohdanecký, et al., ${ }^{14}$ and designated as eq 2:

$$
[\eta]=1.5 \times 10^{-4} M_{w}^{0.77}
$$

The other is developed by Freeman, et al., ${ }^{15}$ and designated as eq 3 :

$$
[\eta]=1.63 \times 10^{-4} M_{w}^{0.766}
$$

Both eq 2 and 3 use $[\eta]$ in THF at $25^{\circ} \mathrm{C}$ while eq 1 uses $[\eta]$ in $\mathrm{THF}$ at $35^{\circ} \mathrm{C}$. This difference may account for $[\eta]$ in eq 1 being slightly larger than that in eq 2 or 3 when compared at the same $M_{w}$.

The number of branch points per unit molecular weight $\lambda$ of branched polymers is expressed as follows ${ }^{16,17}$ for trifunctional branches:

$$
\frac{[\eta]_{\mathrm{b}}}{[\eta]_{1}}=\left[\left(1+\frac{\lambda_{3} M}{7}\right)^{1 / 2}+\frac{4}{9 \pi} \lambda_{3} M\right]^{-0.3}
$$

where $[\eta]_{b}$ is the observed $[\eta]$ of branched polymer, $[\eta]_{1}$ is $[\eta]$ of linear polymer with the same molecular weight $M$ and $\lambda_{3}$ is $\lambda$ of trifunctional branch. Table III presents the results of calculation of $\lambda_{3}$ according to eq 4 for the six fractions in Table II while substituting $M_{w}$ for $M$ and [ $\eta$ ] calculated from eq 1 for $[\eta]_{1}$. All the fractions have a fairly large number of branch points, $1-3$ in every $10^{5}$ of molecular weight or still greater in A-1-15. If the branch is tetra-functional, the number of branch points, $\lambda_{4}$, can be obtained from $\lambda_{3}=$ $2.67 \lambda_{4} \cdot{ }^{17}$

Figure 7 shows the relationship between $\log$ [ $\eta] M_{w}$ and elution volume (universal calibration ${ }^{18}$ ). The indicated molecular weights of the standard polystyrene used are $1.26 \times 10^{6}, 8.6 \times 10^{5}, 4.1 \times 10^{5}$, $1.6 \times 10^{5} .1 .1 \times 10^{5}, 5.1 \times 10^{4}$, and $2.04 \times 10^{4}$, respectively. The $[\eta] M_{w}$ values for polystyrene were derived from $[\eta]=0.99 \times 10^{-4} M_{w}{ }^{0.73} .^{19}$

Grubisic, et al., ${ }^{18}$ demonstrated that, with the

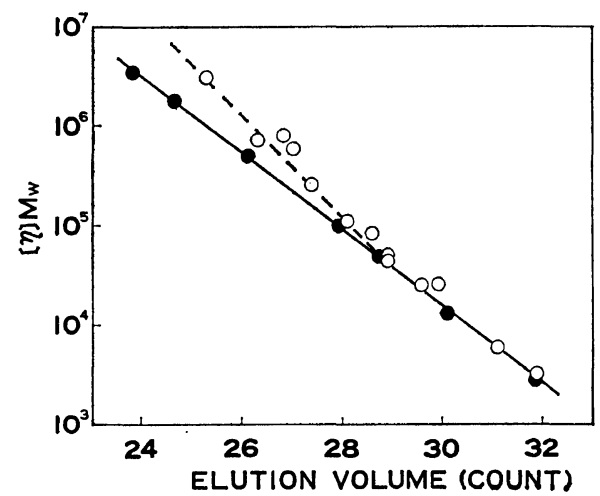

Figure 7. GPC universal calibration: $O$, PVC; $\bullet$ polystyrene.

Table III. The number of branch points per unit molecular weight of PVC fractions

\begin{tabular}{ccc}
\hline Sample & {$[\eta]_{\mathrm{b}} /[\eta]_{1}$} & $\lambda_{3} \times 10^{5}$ \\
\hline A-1-15 & 0.46 & 6.7 \\
A-1-17 & 0.74 & 2.3 \\
B-1-16 & 0.83 & 1.3 \\
B-1-17 & 0.71 & 3.4 \\
B-1-18 & 0.90 & 1.2 \\
B-1-20 & 0.91 & 1.6 \\
\hline
\end{tabular}

Table IV. Comparison of peak elution volume, $V_{\text {peak }}$, and the elution volume at $50 \%$ in cumulative distribution, $V_{50}$

\begin{tabular}{lrrrrr}
\hline \multirow{2}{*}{$\begin{array}{c}\text { Fraction } \\
\text { number }\end{array}$} & \multicolumn{2}{c}{$\mathrm{A}-1$} & & \multicolumn{2}{c}{ B-1 } \\
\cline { 2 - 3 } \cline { 5 - 6 } & $V_{\text {peak }}$ & $V_{\text {50 }}$ & & $V_{\text {peak }}$ & $V_{50}$ \\
\hline 13 & 23.2 & 23.4 & & & \\
14 & 23.8 & 23.9 & & 24.3 & 24.3 \\
15 & 25.3 & 25.1 & & 25.7 & 25.7 \\
16 & 26.2 & 25.9 & & 26.3 & 26.2 \\
17 & 26.8 & 26.4 & & 27.0 & 26.8 \\
18 & 26.7 & 26.7 & & 27.4 & 27.1 \\
19 & 26.8 & 26.9 & & 27.5 & 27.6 \\
20 & 27.0 & 27.2 & & 28.9 & 28.9 \\
21 & 27.7 & 27.8 & & 29.9 & 30.0 \\
22 & 28.6 & 28.6 & & 31.0 & 31.0 \\
23 & 29.5 & 29.6 & & 32.2 & 32.2 \\
24 & 30.7 & 30.8 & & & \\
25 & 31.9 & 31.8 & & \\
\hline
\end{tabular}

use of this universal plot, many kinds of polymers including branched polymers fell on the same curve regardless of the kind of polymers. Based on this finding, it has become possible to determine the degree of branching of branched polymers by 
viscosity-GPC method. ${ }^{20}$ However, in Figure 7, the plot for polystyrene nearly coincides with that for PVC in the low-molecular-weight range, but they deviate from each other in the high-molecularweight range. These similar results have been reported on branched polymers by several workers. $^{20-22}$

Some of the nonsymmetrical chromatograms are found especially for the high-molecular-weight fractions as shown in Figure 2. The elution volume at $50 \%$ in the cumulative GPC chromatogram, $V_{50}$, is given in Table IV compared with the peak elution volume, $V_{\text {peak }}$, for the fractions of A-1 and B-1. For the fractions of Nos. 15-18, $V_{50}$ is less than $V_{\text {peal }}$ by $0.1-0.4$ counts, while $V_{50}$ is nearly equal to $V_{\text {peak }}$ or slightly greater than $V_{\text {peal }}$ for the other fractions. Moreover, if the sample has a log-normal molecular-weight distribution, the molecular weight at $50 \%$ in cumulative distribution, $M_{50}$, is expressed in $\left(M_{w} \cdot M_{n}\right)^{1 / 2}$. So the difference between $\log [\eta] M_{w}$ and $\log [\eta] M_{50}$ is $c a$. 0.1 when $M_{w} / M_{n}=1.6$. As the $M_{w} / M_{n}$ value cannot be much larger than 1.6 for each fraction used in the universal plot as shown in Table II, the difference may be $c a .0 .1$ at the most.

If $M_{50}$ is used instead of $M_{w}$, and also if $V_{50}$ is used instead of $V_{\text {peak }}$. the universal plot for PVC comes near that of polystyrene but both plots seem yet to be in disagreement with each other. Such disagreement seems likely to be due to the branching effect. In order to confirm this, it is neccessary to obtain the fairly narrow-molecular-weightdistribution samples of branched PVC. The method of obtaining such samples from the branched PVC fractions obtained in this work is under investigation.

\section{CONCLUSION}

The fractionation of the sample A and B showed an overload effect. However, the highmolecular-weight fractions of these samples showed fairly small $\left(M_{w} / M_{n}\right)_{\mathrm{GPC}}$ by reducing the injected sample concentration or the collected volume of one fraction. That is, the hydrodynamic-molecular-size distribution of those fractions is fairly narrow in THF used as a preparative scale GPC carrier. Whereas, as shown in A-1-17 or B-1-17, the observed $M_{w} / M_{n}$ values (light scattering and osmometry) of the those fractions are rather larger than $\left(M_{w} / M_{n}\right)_{\mathrm{GPC}}$ owing to the disturbance of long chain branching. Therefore, those fractions are not very suitable as standards for molecular weight but they may be used in discussing the branching of PVC.

Considering that the fairly narrow-hydrodynamic-molecular-size-distribution fractions were obtained as mentioned above, it is concluded that obtaining a fairly narrow-molecular-weightdistribution PVC is possible if the disturbance of the chain branching is eliminated. In sample $\mathrm{C}$, the high-molecular-weight portions containing long chain branches have been cut and so the preparative scale GPC gives fairly narrow-molecular-weight-distribution fractions. They may serve satisfactorily as standard samples in spite of the limitation imposed by the molecular-weight range.

It was observed that the GPC calibration curve for the hydrodynamic volume of PVC agreed with that of polystyrene standards in the low-molecularweight range but not in the high-molecular-weight range where the effect of chain branching was dominant.

Acknowledgment. The authors should like to thank Mr. Akira Wada of Research \& Development Center, Nippon Zeon Co. Ltd. for kindly supplying them with one of the PVC samples.

\section{REFERENCES}

1. K. J. Bombaugh, W. A. Dark, and R. N. King, J. Polym. Sci., Part C, 21, 131 (1968).

2. R. D. Law, ibid., Part A-1, 7, 2097 (1969).

3. S. Hattori and M. Hamashima, Kobunshi Kagaku (Chem. High Polymers), 27, 775 (1970).

4. J.L. Mulder and F.A. Buytenhuys, J. Chromatogr., 51, 459 (1970).

5. A. Barlow, L. Wild, and T. Roberts, ibid., 55, 155 (1971).

6. A. Peyrouset and R. Panaris, J. Appl. Polym. Sci., 16, 315 (1972).

7. Y. Kato, K. Sakane, K. Furukawa, and T. Hashimoto, Kobunshi Kagaku (Chem. High Polymers), 30, 558 (1973).

8. P. G. Montague and F. W. Peaker, J. Polym. Sci., Part C, 43, 277 (1973).

9. Y. Kato, T. Kametani, K. Furukawa, and T. Hashimoto, ibid., Polym. Phys. Ed., 13, 1695 (1975).

10. A. R. Cooper, A. J. Hughes, and J. F. Johnson, J. Appl. Polym. Sci., 19, 435 (1975). 


\section{S. Hattori, H. Endoh, H. Nakahara, T. Kamata, and M. Hamashima}

11. S. Hattori, M. Hamashima, H. Nakahara, and T. Kamata, Kobunshi Ronbunshu (Chem. High Polymers), 34, 503 (1977).

12. National Physical Laboratory (England) data sheet.

13. T. Kamata and H. Nakahara, J. Colloid Interface Sci., 43, 89 (1973).

14. M. Bohdanecký, K. Šolc. P. Kratochvíl, M. Kolínský, M. Ryska, and D. Lim, J. Polym. Sci., Part A-2, 5, 343 (1967).

15. M. Freeman and P. P. Manning, ibid., Part A, 2, 2017 (1964).

16. B. H. Zimm and W. H. Stockmayer, J. Chem. Phys., 17, 1301 (1949).
17. M. Kurata, M. Abe, M. Iwama, and M. Matsushima, Polym. J., 3, 729 (1972).

18. Z. Grubisic, P. Rempp, and H. Benoit, J. Polym. Sci., Part B, 5, 753 (1967),

19. S. Hattori and M. Hamashima, Tokyo Kogyo Shikensho Hokoku (J. National Chem. Lab. Ind.), 71, 457 (1976).

20. M. Kurata, H. Okamoto, M. Iwama, M. Abe, and T. Homma, Polym. J., 3, 739 (1972).

21. T. Kato, A. Itsubo, Y. Yamamoto, T. Fujimoto, and M. Nagasawa, ibid., 7, 123 (1975).

22. M. R. Ambler and D. McIntire, J. Polym. Sci., Polym. Lett. Ed., 13, 589 (1975). 